\title{
Discontinuous Initial Value Problems for Symmetric Hyperbolic Linear Differential Equations*
}

\author{
ROBERT M. LEWIS
}

Communicated by J. KeLLER

\section{INTRODUCTION}

Discontinuous solutions of hyperbolic partial differential equations have often been treated in the mathematical literature. The earlier treatments dealt mainly with second order equations (see e.g. [1], [2]). Recently R. Courant \& P. D. LAx [3] extended the theory to first order linear hyperbolic systems and proved an existence theorem for discontinuous solutions of initial value problems. In this paper we generalize the theory of Courant \& LAX in two respects. First, we consider more general equations. This generalization is not without interest for it permits the inclusion of some important equations of mathematical physics. $\dagger$ Second, we consider initial-boundary value problems rather than pure initial value problems.

In Part II our theory will be applied to the study of asymptotic expansions of solutions of certain partial differential equations.

1. Initial value problems for symmetric hyperbolic equations. Let $u$ be a $k$ component vector function of the $n+1$ variables $x_{0}, x_{1}, \cdots, x_{n}$ (u may be thought of as a row or column matrix, according to the context in which it appears). Let $A^{\nu}$ and $B$ denote $k \times k$ matrices with real entries. We will sometimes denote $x_{0}$ by $t$ (since in physical problems it may represent time) and the "space variables" $x_{1}, \cdots, x_{n}$ by $x$. The matrices $A^{\nu}$ and $B$ are taken to be

*The research reported in this article has been sponsored by the Department of Defense under Contract No. DA49-170-sc-2253.

$\dagger$ That the present theory is directly applicable to Maxwell's equations will be demonstrated in Asymptotic Expansion of Steady-State Solutions of Symmetric Hyperbolic Linear Differential Equations. This paper appears in the present issue and will be referred to as Part II. 
smooth $^{*}$ functions of $x$ and $t$ in the half-space $t \geqq 0$. We define a first order linear differential operator

$$
L u=\sum_{\nu=0}^{n} A^{\nu} u_{x_{\nu}}+B u \quad\left(u_{x_{\nu}}=\frac{\partial u}{\partial x_{\nu}}\right) .
$$

The operator $L$ will be taken to be symmetric hyperbolic, i.e., the matrices $A^{\text {" }}$ are assumed to be symmetric, and the matrix $A^{0}$ to be positive-definite. These definitions are discussed in [4] and [5]. Without loss of generality we shall take $A^{0}=I$, where $I$ is the identity matrix. This is possible because the positive definite matrix $A^{0}$ may be written as $A^{0}=T^{\prime} T$, where $T$ is a non-singular matrix. Then the transformation of dependent variables $T u=v$ transforms $L$ into a symmetric hyperbolic operator on $v$ in which the coefficient of $v_{t}$ is $I$.

Linear symmetric hyperbolic differential equations have been extensively studied in recent years [4], [5]. One result of this study is the following existence, uniqueness, and differentiability theorem:

Theorem 1. Let $L$ be a symmetric hyperbolic operator with smooth coefficients, $\phi(t, x)$ a smooth function defined in the half-space $t \geqq 0$, and $g(x)$ a smooth initial function defined in the entire $x$-space. Then the initial value problem

$$
L u=\phi, \quad u(0, x)=g(x)
$$

has a unique smooth solution $u(t, x)$ defined in the entire half-space. The value of the solution $u$ at a given point depends on the values of the coefficients, and of the functions $\phi$ and $g$, only in a finite region. $\dagger$

Since it is our object to extend this theorem to discontinuous functions, we must introduce the notion of a "weak solution." For this purpose we consider a space $S$ of smooth testing functions $\Omega(t, x)$ with compact support. More precisely, $\mathcal{S}$ will be the collection of all smooth $k$ component vector functions $\Omega$ each of which vanishes identically outside some compact region $R$ which depends upon $\Omega$ and lies in the half space $t>0$. For any $u$ in $C^{1}$, the set of functions with continuous first derivatives, and any $\Omega$ in $\delta$ we have the identity

$$
\iint \Omega L u d x d t=\iint u M \Omega d x d t
$$

Here $M$, the adjoint of $L$, is defined by

$$
M \Omega=-\sum_{\nu=0}^{n}\left(A^{\nu} \Omega\right)_{x_{\nu}}+B^{\prime} \Omega .
$$

\footnotetext{
*Throughout this paper, the term "smooth" will be used to describe functions which have continuous derivatives of sufficiently high order for the purpose at hand. The precise order can always be obtained by careful examination of the argument, but will usually be ignored.

$\uparrow$ This region is the "backward characteristic cone." We will not be concerned with its precise delineation.
} 
$B^{\prime}$ denotes the transpose of the matrix $B$. The integration in (2) extends over the half-space $t \geqq 0$. The identity is obtained simply by integration by parts, the boundary terms vanishing because $\Omega$ has compact support. If now we have $L u=0$, it follows trivially that

$$
\iint u M \Omega d x d t=0 \text { for all } \Omega \text { in } \delta .
$$

Conversely, if $u$ is in $C^{1}$ and satisfies (4) it follows that $L u=0$ in the half-space $t>0$.

We may, however, consider more general classes of functions than $C^{1}$. Accordingly, we introduce the class $\nVdash$ of piece-wise smooth functions: For each vector function $u$ in $\Re$, defined in the region of interest $\mathbb{D}$ (here $\mathscr{D}$ is the half-space $t \geqq 0$ ), there are a finite number of smooth hypersurfaces which divide $D$ into subregions $\mathscr{D}_{\nu}$ such that the first derivatives of $u$ can be made continuous in the closure of each $\mathscr{D}_{\nu}$ by appropriate definition of the derivative on the boundary.* If a function $u$ in $\Re$ satisfies (4) we say that $u$ is a weak solution of the equation $L u=0$. If in addition $u(0, x)=g(x)$, we say that $u$ is a weak solution of the initial value problem

$$
L u=0, \quad u(0, x)=g(x) .
$$

We will now analyze the discontinuities and prove the existence of weak solutions of the problem (5) for appropriate discontinuous functions $g$.

2. The transport equations. Consider a function $u$ which is a solution of (4) and which is smooth everywhere except on a smooth hypersurface $C$ whose equation is $\phi^{0}(t, x)=0 . u$ and all of its derivatives are assumed to have finite jump discontinuities across $C$. We introduce a regular transformation $y_{i}=$ $\phi^{i}\left(x_{0}, \cdots, x_{n}\right), j=0,1, \cdots, n$, which utilizes $C$ as a coordinate surface. The equation of $C$ becomes $y_{0}=0$. In the new coordinates we have

$$
L u=\sum_{j=0}^{n} H^{j} u_{y_{i}}+B u .
$$

Here we have introduced the matrices $H^{i}$ defined by

$$
H^{i}=\sum_{\nu=0}^{n} \phi_{x}^{j} A^{\nu}, \quad j=0,1, \cdots, n .
$$

Let $D_{1}$ and $D_{2}$ denote regions on either side of $C$ and let $u=u^{1}+u^{2}$ where $u^{1} \equiv 0$ in $\mathscr{D}_{2}$ and $u^{2} \equiv 0$ in $\mathscr{D}_{1}$. By Gauss' theorem

$$
\iint\left[-u^{1}\left(A^{\nu} \Omega\right)_{x_{v}}\right] d x d t=\iint \Omega A^{\nu} u_{x_{\nu}}^{1} d x d t-\int_{C}\left(\Omega A^{\nu} u^{1}\right) \alpha \phi_{x_{\nu}}^{0} d S .
$$

*Then the discontinuities of $u$ and its first derivatives will be jump discontinuities across the hypersurfaces. 
Here $\alpha$ is a scalar factor so chosen that $\left(\phi_{x_{0}}^{0}, \cdots, \phi_{x_{n}}^{0}\right) \alpha$ is a unit vector pointing in the required direction. It follows that

$$
\iint u^{1} M \Omega d x d t=\iint \Omega L u^{1} d x d t-\int_{C} \sum_{\nu=0}^{n}\left(\Omega A^{\nu} u^{1}\right) \alpha \phi_{x_{\nu}}^{0} d S .
$$

By adding (9) to the corresponding equation for $u^{2}$, we obtain an equation in which the double integrals vanish because of (4) and the fact that $L u=0$ everywhere except on $C$. We are left with

$$
\int_{C} \sum_{\nu=0}^{n} \Omega A^{\nu}[u] \alpha \phi_{x}^{0} d S=\int_{C} \Omega H^{0}[u] \alpha d S=0 .
$$

The symbol $[u]=u^{2}-u^{1}$ denotes the jump in $u$ across $C$. Since the components of $\Omega$ are arbitrary it follows that

$$
H^{0}[u]=0 .
$$

If the vector $[u]$ is not zero, the matrix $H^{0}$ must be singular, i.e.,

$$
\operatorname{det}\left(\sum_{\nu=0}^{n} \phi_{x_{\nu}}^{0} A^{\nu}\right)=0 \text {. }
$$

But if $\phi^{0}$ is a solution of (12), the hypersurface $\phi^{0}=0$ is by definition a characteristic hypersurface for the operator $L$. Thus if $u$ is discontinuous across a hypersurface $C$, then $C$ must be characteristic. We shall see later that the same conclusion follows if any derivative of $u$ is discontinuous across $C$.

At this point we impose an important condition on the class of operators $L$ under consideration, the

Multiplicity Condition: If $P(t, x, z)=\sum_{\nu=1}^{n} z_{\nu} A^{\nu}$, where the $z_{\nu}$ are real numbers not all zero, then the multiplicities of the eigenvalues of the matrix $P$ are independent of $t, x$, and $z=\left(z_{1}, \cdots, z_{n}\right) *$

The multiplicity condition assures us that certain eigenvalues $\alpha_{1}, \cdots, \alpha_{a}$ of $H^{0}$ are identically zero on $C$ and the others are non-zero. ${ }^{* *}$ Let $R^{v}$ denote the associated orthonormal eigenvectors. From (11), $[u]$ is in the null space of $H^{0}$, and hence can be expanded in terms of the null eigenvectors (i.e., those associated with zero eigenvalues):

$$
[u]=\sum_{m=1}^{q} \sigma_{m}^{0} R^{m}
$$

*In the work of Couranx \& LAx [3], the eigenvalues are required to be distinct (i.e., of multiplicity one). For Maxwell's equations $P$ has three eigenvalues, each of multiplicity two (see Part II).

**It follows from the lemma of Appendix 1 that the eigenvalues of $H^{0}$ are continuous functions of position on $C$. If now $\alpha_{1}, \cdots, \alpha_{q}$ are identically zero in a region $D_{1}$ on the hypersurface $C$, and another set $\beta_{1}, \cdots, \beta_{p}$ were identically zero in a neighboring region $\mathrm{D}_{2}$ on $C$, then by continuity both sets would be zero on the common boundary of $\mathscr{D}_{1}$ and $\mathscr{D}_{2}$ in violation of the multiplicity condition. 
The $\sigma_{m}^{0}$ are scalars. Since $L u=0$ on both sides of $\phi_{0}=0$,

$$
\sum_{i=0}^{n} H^{i}\left[u_{y_{j}}\right]+B[u]=0 .
$$

For $j \neq 0, u_{y_{j}}$ is a derivative tangential to $C$, so $\left[u_{y_{i}}\right]=[u]_{y_{i}}$. Thus

$$
H^{0}\left[u_{y_{0}}\right]+\sum_{j=1}^{n} H^{j}[u]_{y_{i}}+B[u]=0 .
$$

Since $H^{0} R^{\mu}=R^{\mu} H^{0}=0, \mu=1, \cdots, q$, multiplication of (15) by $R^{\mu}$ yields

$$
\sum_{j=1}^{n} R^{\mu} H^{j}[u]_{y_{i}}+R^{\mu} B[u]=0, \quad \mu=1, \cdots, q .
$$

Substituting (13) into (16),

$$
\begin{aligned}
\sum_{j=1}^{n} \sum_{m=1}^{q} R^{\mu} H^{i} R^{m}\left(\sigma_{m}^{0}\right)_{y_{i}}+\sum_{i=1}^{n} \sum_{m=1}^{q} R^{\mu} H^{j} R_{y_{i}}^{m} \sigma_{m}^{0}+\sum_{m=1}^{q} R^{\mu} B R^{m} \sigma_{m}^{0} & =0, \\
\mu & =1, \cdots, q .^{*}
\end{aligned}
$$

Equation (17) is a first order linear system of partial differential equations for the scalar coefficients $\sigma_{m}^{0}$ in the expansion of $[u]$. It is called the transport equation of order zero. Before discussing it further, we will derive the corresponding equations for the discontinuities in the derivatives of $u$. From (6) we have for $r=1,2, \cdots$

$$
\frac{\partial^{r}}{\partial y_{0}^{r}} L u=\sum_{j=0}^{n} H^{j} u_{y_{0} r_{y_{j}}}+B u_{y_{0} r}+r \sum_{j=0}^{n} H_{y_{0}}^{i} u_{y_{0}(r-1) y_{j}}+N^{(r-1)} u .
$$

Here the operator $N^{(r-1)}$ involves derivatives of $u$ of order $r-1$ or less, and we have used the abbreviation

$$
u_{y_{0} r_{j}}=\frac{\partial^{(r+1)} u}{\partial y_{0}^{r} \partial y_{i}}
$$

Since $L u \equiv 0$ on both sides of $C$,

$$
\begin{aligned}
H^{0}\left[u_{y_{0}(r+1)}\right] & +\sum_{j=1}^{n} H^{i}\left[u_{y_{0}}\right]_{y_{i}}+\left(B+r H_{y_{0}}^{0}\right)\left[u_{y_{0} r}\right] \\
& +r \sum_{j=1}^{n} H_{y_{0}}^{i}\left[u_{y_{0}(r-1)}\right]_{y_{i}}+\left[N^{(r-1)} u\right]=0 .
\end{aligned}
$$

If all derivatives of $u$ of order less than $r+1$ are continuous across $C$, then $H^{0}\left[u_{y_{0}(r+1)}\right]=0$. If, now, some $(r+1)^{\mathrm{st}}$ derivative has a non-zero jump, then $\left[u_{y_{0}(r+1)}\right] \neq 0 \dagger$ and $H^{0}$ is singular. Thus we may assert that if $u$ or any of its

*A derivative of an eigenvector appears in (17). The lemma of Appendix 1 shows that the eigenvalues and eigenvectors of $H_{0}$ are smooth functions.

†Any $(r+1)^{\text {st }}$ derivative can be expressed as a linear combination of $u_{y_{0}(r+1)}$ and tangential derivatives of $r^{\text {th }}$ derivatives of $u$. Since all $r^{\text {th }}$ derivatives are continuous, their tangential derivatives are continuous. Hence $u_{y_{0}(r+1)}$ must be discontinuous. 
derivatives has a jump discontinuity across $C$, then $C$ is a characteristic surface. Suppose now $\left[u_{y_{0}}\right.$ ] is known for $s=0,1, \cdots, r-1$. Then the jumps in all derivatives of $u$ of order $r-1$ or less are known, and hence $\left[N^{(r-1)} u\right]$ is known. By substituting $r$ for $r+1$, we can rewrite (19) in the form

$$
H^{0}\left[u_{y_{0} r}\right]+\sum_{i=1}^{n} H^{j}\left[u_{y_{0}(r-1)}\right]_{y_{j}}+\left[P^{(r-1)} u\right]=0 .
$$

Here $\left[P^{(r-1)} u\right]$ involves derivatives of $u$ of order $r-1$ or less, so it is known. We now expand $\left[u_{y_{0}}\right]$ in terms of all the eigenvectors of $H^{0}$ :

$$
\left[u_{y_{\circ}}\right]=\sum_{m=1}^{k} \sigma_{m}^{r} R^{m}
$$

From (20) and (21) we have

$$
H^{0}\left[u_{y_{0}}\right]=\sum_{m=q+1}^{k} \alpha_{m} \sigma_{m}^{r} R^{m}=-\sum_{j=1}^{n} H^{j}\left[u_{y_{0}(r-1)}\right]_{y_{j}}-\left[P^{(r-1)} u\right] .
$$

Multiplying by $R^{s}$,

$$
\sigma_{s}^{r}=-\frac{1}{\alpha_{s}}\left\{\sum_{j=1}^{n} R^{s} H^{j}\left[u_{y_{0}(r-1)}\right]_{y_{i}}+R^{s}\left[P^{(r-1)} u\right]\right\}, \quad s=q+1, \cdots, k .
$$

Thus (23) gives the coefficients of the non-null eigenvectors in the expansion of $\left[u_{y_{0}}\right.$ ] $]$ in terms of known functions. To obtain the other coefficients, we multiply (19) by a null eigenvector:

$$
\sum_{i=1}^{n} R^{\mu} H^{i}\left[u_{y_{0}}\right]_{y_{i}}+R^{\mu}\left(B+r H_{y_{0}}^{0}\right)\left[u_{y_{0}}\right]=R^{\mu}\left[T^{(r-1)} u\right], \quad \mu=1, \cdots, q .
$$

$\left[T^{(r-1)} u\right.$ ] involves derivatives of $u$ of order less than $r$, hence by assumption is known. Upon substituting (21) into (24), we obtain

$$
\begin{aligned}
& \sum_{i=1}^{n} \sum_{m=1}^{q} R^{\mu} H^{j} R^{m}\left(\sigma_{m}^{r}\right)_{y_{i}}+\sum_{j=1}^{n} \sum_{m=1}^{q} R^{\mu} H^{i} R_{y_{i}}^{m} \sigma_{m}^{r} \\
&+\sum_{m=1}^{q} R^{\mu}\left(B+r H_{y_{0}}^{0}\right) R^{m} \sigma_{m}^{r}=g_{\mu}^{r}, \\
& \mu=1, \cdots, q .
\end{aligned}
$$

The symbol $g_{\mu}^{r}$ is defined by

$$
g_{\mu}^{r}=R^{\mu}\left[T^{(r-1)} u\right]-\sum_{j=1}^{n} R^{\mu} H^{j}\left[\sum_{s=q+1}^{k} \sigma_{s}^{r} R^{s}\right]_{y_{i}}-R^{\mu}\left(B+r H_{\nu_{0}}^{0}\right) \sum_{s=q+1}^{k} \sigma_{s}^{r} R^{s} .
$$

The $g_{\mu}^{r}$ are known functions, the $\sigma_{s}^{r}$ being given by (23) in terms of lower order jumps. Let us establish the convention that quantities with negative superscripts are zero; then since $\sigma_{s}^{0}=0$ for $s>q, g_{\mu}^{0}=0$ and (25) contains (17) as the special case $r=0$. We rewrite (25) as 


$$
\begin{aligned}
\sum_{j=1}^{n} \sum_{m=1}^{q} R^{\mu} H^{i} R^{m}\left(\sigma_{m}^{r}\right)_{y_{j}}+\sum_{m=1}^{a} \tau_{\mu m}^{r} \sigma_{m}^{r}=g_{\mu}^{r}, \quad \mu=1, \cdots, q, & \\
r & =0,1, \cdots,
\end{aligned}
$$

where

$$
\tau_{\mu m}^{r}=\sum_{i=1}^{n} R^{\mu} H^{i} R_{y_{j}}^{m}+R^{\mu}\left(B+r H_{y_{0}}^{0}\right) R^{m} .
$$

It can be shown that (27) is for each $r$ a symmetric hyperbolic system. Therefore we could apply Theorem 1 to assert the existence and uniqueness of its solution. However we can improve on this approach: We shall show that (27) reduces to a system of ordinary differential equations. ${ }^{*}$ To see this, we shall prove the following

Lemma: Let $R_{0}^{1}, \cdots, R_{0}^{a}$ be orthonormal eigenvectors associated with the same eigenvalue $\lambda_{0}$ of the matrix $P=\sum_{v=1}^{n} z_{\nu} A^{\nu}$ ( $z_{\nu}$ real). Then for each $j$

$$
R_{0}^{\mu} A^{i} R_{0}^{m}=c_{i} \delta_{\mu m}
$$

(i.e., $R_{0}^{\mu} A^{i} R_{1}^{m}=0$ if $\mu \neq m$, and is independent of $\mu$ if $\mu=m$ ).

Proof: For any $j$ let $M(\zeta)=P+\zeta A^{i}$. Let $R^{1}(\zeta), \cdots, R^{q}(\zeta)$ be orthonormal eigenvectors of $M$ corresponding to the eigenvalue $\lambda(\zeta)$ where $\lambda(0)=\lambda_{0}$ and $R^{\nu}(0)=R_{0}^{\nu}$. From the multiplicity condition, it follows by means of the lemma of Appendix 1 that the $R^{\nu}(\zeta)$ and $\lambda(\zeta)$ are analytic functions in a neighborhood of $\zeta=0$.

Let $R_{1}^{m}$ and $\lambda_{1}$ denote the derivatives with respect to $\zeta$ evaluated at $\zeta=0$ of $R^{m}(\zeta)$ and $\lambda(\zeta)$ respectively. Differentiating the equation $M R^{m}=\lambda R^{m}$ we obtain

$$
A^{i} R_{0}^{m}+P R_{1}^{m}=\lambda_{1} R_{0}^{m}+\lambda_{0} R_{1}^{m},
$$

and, multiplying on the left by $R_{0}^{\mu}$,

$$
R_{0}^{\mu} A^{i} R_{0}^{m}=R_{0}^{\mu}\left(\lambda_{0}-P\right) R_{1}^{m}+\lambda_{1} R_{0}^{\mu} R_{0}^{m}=\lambda_{1} \delta_{\mu m} .
$$

This completes the proof of the lemma.

Since the matrices $H^{i}$ are just linear combinations of the matrices $A^{j}$ it follows from the lemma that

(30) $R^{\mu} H^{i} R^{m}=0 \quad$ if $\quad \mu \neq m, \quad R^{\mu} H^{i} R^{\mu}=R^{1} H^{j} R^{1} \quad$ for $\quad \mu, m=1, \cdots, q$.

Thus (27) may be rewritten

$$
\sum_{j=1}^{n} R^{1} H^{i} R^{1}\left(\sigma_{\mu}^{r}\right)_{y_{i}}+\sum_{m=1}^{q} \tau_{\mu m}^{r} \sigma_{m}^{r}=g_{\mu}^{r}, \quad \mu=1, \cdots, q, \quad r=0,1, \cdots .
$$

*For the very useful (and far from obvious) observation, we are indebted to P. UNGAR. 
Expanding $H^{i}$ as in (7) yields

$$
\begin{aligned}
\sum_{i=1}^{n} R^{1} H^{i} R^{1}\left(\sigma_{\mu}^{r}\right)_{y_{i}} & =\sum_{i=1}^{n} \sum_{\nu=0}^{n} \phi_{x_{\nu}}^{i} R^{1} A^{\nu} R^{1}\left(\sigma_{\mu}^{r}\right)_{y_{j}} \\
& =\sum_{\nu=0}^{n} R^{1} A^{\nu} R^{1} \sum_{j=1}^{n}\left(\sigma_{\mu}^{r}\right)_{y_{j}} \frac{\partial y_{i}}{\partial x_{\nu}}=\sum_{\nu=0}^{n} R^{1} A^{\nu} R^{1}\left(\sigma_{\mu}^{r}\right)_{x_{\nu}} .
\end{aligned}
$$

Here we have used the fact that $\left(\sigma_{\mu}^{r}\right)_{y_{0}}=0$. Thus (31) becomes

$$
\sum_{\nu=0}^{n} R^{1} A^{\nu} R^{1}\left(\sigma_{\mu}^{r}\right)_{x_{\nu}}+\sum_{m=1}^{q} \tau_{\mu m}^{r} \sigma_{m}^{r}=g_{\mu}^{r}, \quad \mu=1, \cdots, q, \quad r=0,1, \cdots .
$$

We now consider a family of curves in the $t x$-space defined parametrically by $x_{\nu}=x_{\nu}(s), \nu=0,1, \cdots, n$, where

$$
\dot{x}_{\nu}=\frac{d x_{\nu}}{d s}=R^{1} A^{\nu} R^{1}, \quad \nu=0,1, \cdots, n .
$$

Since $\dot{x}_{0}=\dot{t}=1$, we may identify $t$ with $s$ (up to an additive constant on each curve). Then (33) may be interpreted as ordinary differential equations along these curves, for

$$
\sum_{\nu=0}^{n} R^{1} A^{\nu} R^{1}\left(\sigma_{\mu}^{r}\right)_{x_{\nu}}=\sum_{\nu=1}^{n} \dot{x}_{\nu}\left(\sigma_{\mu}^{r}\right)_{x_{\nu}}=\dot{\sigma}_{\mu}^{r}
$$

Hence

$$
\dot{\sigma}_{\mu}^{r}+\sum_{m=1}^{q} \tau_{\mu m}^{r} \sigma_{m}^{r}=g_{\mu}^{r}, \quad \mu=1, \cdots, q, \quad r=0,1, \cdots .
$$

We observe that $\sum_{\nu=0}^{n} \dot{x}_{\nu} \phi_{x_{\nu}}^{0}=R^{1} H^{0} R^{1}=0$ on $\phi^{0}=0$. Hence the curves which we have introduced are tangential to the characteristic surface $\phi^{0}=0$. It can be shown that these curves, which we shall refer to as rays, are identical with the characteristic curves of the single first order partial differential equation (12) for $\phi^{0}$. As such they are sometimes referred to as the bicharacteristics of the operator $L$. From the theory of first order partial differential equations, we know that the characteristic surface $\phi^{0}=0$ is generated by an $n-1$ parameter family of rays.

Thus, for each fixed $r$, (35) is a system of $q$ linear ordinary differential equations along the rays. Examination of the foregoing argument shows that the coefficients $\tau_{\mu m}^{r}$ and $g_{\mu}^{r}$ will be smooth functions provided the hypersurface $C$ is smooth and the $\sigma_{m}^{\nu}$ are smooth for $\nu<r$. Given appropriate initial values (these will be derived in the next section), we may then assert that there exists a unique smooth solution of (35). Thus the system of differential equations (35) and algebraic equations (23) can, in principle, be solved recursively for $r=0,1, \cdots, r_{1}$, and for $r_{1}$ as large as we wish. These equations will be referred to as the transport equations (of order $r$ ).

Remark. The higher transport equations discussed above determine the 
discontinuities in derivatives of $u$ normal to the characteristic surface $C$. In the special case that $C$ never has a tangent plane parallel to the $t$-axis, we could instead consider derivatives in the t-direction. If, in addition, the coefficients $A^{\nu}, B$ are independent of $t$ this leads to a considerable simplification of the equations. A simplified analysis based on these assumptions is carried out in Part II, Section 6.

3. Initial conditions for the transport equations. In the previous section we were concerned with a single characteristic surface $C$ and its associated transport equations. Since, in what follows, we shall have to be concerned with several distinct characteristic surfaces $C^{(p)}$, we will use an additional index $(p)$ on various quantities.

Let $\Gamma$ be a given smooth $n-1$ dimensional manifold in the initial hyperplane $t=0$. Let $g(x)$ be smooth on both sides of $\Gamma$, while $g$ and its derivatives suffer jump discontinuities across $\Gamma$. Let $\gamma_{1}, \cdots, \gamma_{n}$ be components of the unit normal to $\Gamma$, and let $\phi^{(p)}=\phi^{0(p)}(t, x)=0$ be the equation of a characteristic surface $C^{(p)}$ whose intersection with the hyperplane $t=0$ is $\Gamma$. Then $\phi_{x_{\nu}}^{(p)}=\rho \gamma_{\nu}(\nu=$ $1, \cdots, n)$ on $\mathrm{\Gamma}$, where $\rho$ is a proportionality factor. ${ }^{*} \phi_{x_{0}}^{(p)}=\phi_{l}^{(p)}$ is a solution of

$$
\operatorname{det}\left(\sum_{\nu=0}^{n} \phi_{x_{\nu}}^{(p)} A^{\nu}\right)=\operatorname{det} H^{0(p)}=\operatorname{det}\left(\phi_{t}^{(p)}+\rho E\right)=0 \text { on } \Gamma
$$

where

$$
E=\sum_{\nu=1}^{n} \gamma_{\nu} A^{\nu}
$$

Let $R^{i}, j=1, \cdots, k$, denote orthonormal eigenvectors of $E$ (hence of $H^{0(p)}$ on $\Gamma)$ and $\lambda_{i}$ the corresponding eigenvalues. Then the eigenvalues of $H^{0(p)}$ are $\phi_{t}^{(p)}+\rho \lambda_{j}$. By virtue of (36), for each $p$ and for some $j$,

$$
\phi_{t}^{(p)}+\rho \lambda_{j}=0 .
$$

There will be as many distinct characteristic surfaces $C^{(p)}$ intersecting $\Gamma$ as there are distinct solutions $\phi_{t}^{(p)}$ of (38), hence as many as there are distinct eigenvalues of $E$. The multiplicity condition assures us that the number of these distinct characteristic surfaces $C^{(p)}$ is the same at every point of $\Gamma$. On each such $C^{(p)}$ a different set of eigenvectors is null for $H^{0(p)}$ (those $R^{j}$ for which $\lambda_{i}$ satisfies (38)), but the totality of null eigenvectors for all the surfaces $C^{(p)}$ is the totality of the eigenvectors of $E$.

Let []$^{p},[]^{\Gamma}$ denote jumps across $C^{(p)}$ and $\Gamma$ respectively. Since the eigenvectors $R^{j}$ form a complete set the jump in initial values of $u$ across $\Gamma$ has the unique decomposition

\footnotetext{
*Geometrically, this is the condition that the projection onto the hyperplane $t=0$ of a vector normal to $C^{(p)}$ at a point on $\Gamma$ shall be parallel to the normal vector $\left(\gamma_{1}, \cdots, \gamma_{n}\right)$ of $\Gamma$. The condition is equivalent to the so-called "strip manifold condition" in the theory of first order partial differential equations. See e.g., [1], Chapter II.
} 


$$
[u]^{\Gamma}=[g]^{\Gamma}=\sum_{m=1}^{k} \sigma_{0 m}^{0} R^{m} .
$$

We rewrite (13), including the additional index $p$ :

$$
[u]^{p}=\sum_{m=1}^{k} \sigma_{p m}^{0} R^{m}
$$

Here $\sigma_{p m}^{0}=0$ if $R^{m}$ is not a null eigenvector of $H^{0(p)}$. The initial conditions for the $\sigma_{p m}^{0}$ are obtained from the fact that

$$
\sum_{p}[u]^{p}=[u]^{\mathrm{r}} \text { on } \Gamma \text {. }
$$

Multiplying by any eigenvector $R^{\circ}$,

$$
\sum_{p} \sigma_{p v}^{0}=\sigma_{0 v}^{0} \text { on } \Gamma \text {. }
$$

But, for every $v, R^{v}$ is a null eigenvector of $H^{0}$ on exactly one of the characteristic surfaces, say $C^{(a)}$. Hence $\sigma_{p v}^{0}=0$ if $p \neq q$ and (42) becomes

$$
\sigma_{a v}^{0}=\sigma_{0 v}^{0} \text { on } \Gamma \text {. }
$$

Thus (43) determines the initial values of all the $\sigma_{p m}^{0}$.

To obtain the initial conditions for the higher transport equations, we suppose now that $\left[u_{y_{0} \rho}\right]^{p}$ have been determined on $\Gamma$ for all $p$ and for $\rho=0,1, \cdots, r-1$. Then the jumps in all derivatives of order less than $r$ can be calculated. (Note that we have a different variable $y_{0}$ for each of the $C^{(p)}$ and the derivative $\partial / \partial y_{0}$ is directed normally to $C^{(p)}$.) For each characteristic surface $C^{(p)}$, it is convenient to introduce two directions of differentiation at each point of $\Gamma: \partial / \partial N$ (normal to $\Gamma$ in the plane $t=0$ ) and $\partial / \partial s$ (normal to $\Gamma$ and tangential to $\left.C^{(p)}\right)$. The corresponding directions are indicated in Fig. 1. Since the three directions $\left(y_{0}, N, s\right)$ are all normal to $\Gamma$ they are linearly dependent, hence we may expand $u_{N^{r}}$ as

$$
u_{N^{r}}=\sum_{\nu=0}^{r} e_{\nu}^{p} u_{y_{0}(r-v)_{s}} .
$$

Here the $e_{v}^{p}$ are known angle factors, and $e_{0}^{p} \neq 0$ since the plane $t=0$ is noncharacteristic.* From (44)

$$
\left[u_{N^{r}}\right]^{p}=e_{0}^{p}\left[u_{y_{0} r}\right]^{p}+\sum_{\nu=1}^{r} e_{\nu\lfloor}^{p}\left[u_{y_{0}(r-p)}\right]_{s \nu^{\nu}}^{p} .
$$

By (45), $\left[u_{N_{r}}\right]^{p}$ and $\left[u_{y_{0}}\right]^{p}$ determine each other uniquely. As before, we rewrite (21) as

$$
\left[u_{y_{0} r}\right]^{p}=\sum_{m=1}^{k} \sigma_{p m}^{r} R^{m}
$$

\footnotetext{
${ }^{*}$ A characteristic surface can never have a tangent plane parallel to the plane $t=0$. This follows from (12).
} 


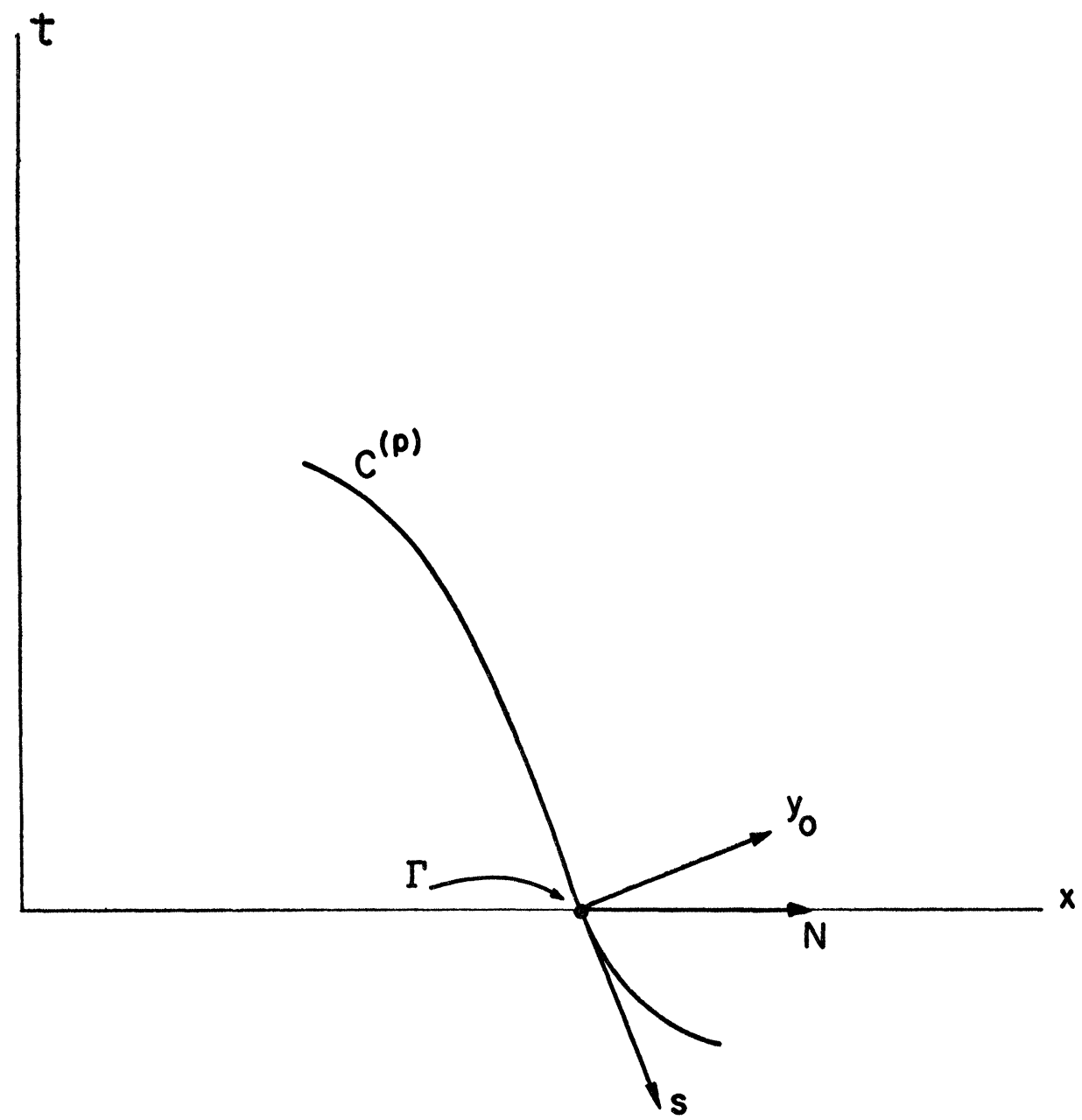

Figure 1

Relative orientations of the directions $y_{0}, N, s$ on the initial plane.

Let

$$
\left[u_{N^{r}}\right]^{p}=\sum_{m=1}^{k} \beta_{p m}^{r} R^{m}
$$

Then from (45)

$$
\beta_{p_{\mu}}^{r}=R^{\mu}\left[u_{N^{r}}\right]^{p}=e_{0}^{p} \sigma_{p \mu}^{r}+\gamma_{p \mu}^{r}
$$

where

$$
\gamma_{p \mu}^{r}=R^{\mu} \sum_{\nu=1}^{r} e_{\nu}^{p}\left[u_{y_{0}(r-v)}\right]_{s^{\nu}}^{p}
$$


By (48), the $\beta_{p \mu}^{r}$ and $\sigma_{p \mu}^{r}$ determine each other uniquely in terms of the $\gamma_{p \mu}^{r}$ which we may assume to be known. The initial conditions for the $\sigma_{p \mu}^{r}$ are obtained from the fact that

$$
\sum_{p}\left[u_{N^{r}}\right]^{p}=\left[u_{N^{r}}\right]^{\mathrm{\Gamma}}=\left[g_{N^{r}}\right]^{\mathrm{r}} \text { on } \Gamma .
$$

As before, we have

$$
\left[u_{N^{r}}\right]^{\mathrm{r}}=\left[g_{N^{r}}\right]^{\mathrm{r}}=\sum_{m=1}^{k} \sigma_{0 m}^{r} R^{m} .
$$

Multiplying by any $R^{v},(50)$ becomes

$$
\sigma_{0 v}^{r}=\sum_{p} \beta_{p v}^{r}=\sum_{p}\left(e_{0}^{p} \sigma_{p v}^{r}+\gamma_{p v}^{r}\right) \quad \text { on } \Gamma .
$$

The $\sigma_{p v}^{r}$ are predetermined by (23) except when $R^{v}$ is a null eigenvector of $H^{0(p)}$. This occurs on exactly one of the characteristic surfaces, say $C^{(q)}$. Thus $\sigma_{a v}^{r}$ is not determined by (23) and its initial values are given by

$$
e_{0}^{a} \sigma_{q v}^{r}+\gamma_{a v}^{r}=\sigma_{0 v}^{r}-\sum_{p \neq a}\left(e_{0}^{p} \sigma_{p v}^{r}+\gamma_{p v}^{r}\right) \quad \text { on } \Gamma \text {. }
$$

The initial values of the $\sigma_{a v}^{r}$ will be smooth functions along $\Gamma$ provided the $\sigma_{0 v}^{r}$ are smooth, i.e., provided the $\left[g_{N^{r}}\right]^{\Gamma}$ are smooth. But this is guaranteed by our assumption that $g$ is smooth on both sides of $\Gamma$. It follows that the solutions of the transport equations will be smooth functions of the parameters along $\Gamma$, hence smooth functions of position on the characteristic surface (i.e., not only in the ray direction).

4. Existence and uniqueness theorem for the discontinuous initial value problem. For the proof of the theorem which we are about to present, it is necessary that we be able to solve the transport equations (plus initial conditions) along the characteristic surfaces $C^{(p)}$ emanating from the manifold $\Gamma$. It is clear from the foregoing that this can be done in the region $0 \leqq t<t_{1}$, where $t_{1}$ is the smallest value of $t$ for which one of the hypersurface $C^{(p)}$ has a singularity. Singularities of characteristic surfaces bearing non-zero jumps will be referred to as caustics. Whether or not caustics appear in a given initial value problem cannot be determined solely by examining the solutions $\phi$ of the single first order partial differential equation (12) whose solution surfaces $\phi=0$ contain $\Gamma$; for it is possible that the function $g(x)$ may be such that although the characteristic surface $C^{(p)}$ emanating from $\Gamma$ has a singularity, that surface does not play a role, the corresponding initial values for the transport equations being all zero. It is convenient to formulate the following

Caustic Condition: The problem is such that the characteristic surfaces across which $u$ and its derivatives have non-zero jumps are free of caustics.

We may now state 
Theorem 2: Given the initial value problem

$$
\begin{gathered}
L u=0, \\
u(0, x)=g(x),
\end{gathered}
$$

where $L$ is a symmetric hyperbolic operator with smooth coefficients which satisfies the multiplicity condition and $g(x)$ is smooth on either side of a smooth $n-1$ dimensional manifold $\Gamma$ in the plane $t=0$, while $g$ and its derivatives have jump discontinuities across $\Gamma$, there exists one and only one weak solution $u$ in the class $\mathfrak{K}$ for $t$ in the interval $0 \leqq t \leqq t_{1} .^{*} u$ is smooth for all $x$ and for $t$ in that interval except on the characteristic surfaces emanating from $\Gamma$. Across these surfaces $u$ and its derivatives have jump discontinuities which satisfy the transport equations. If the caustic condition is satisfied we may take $t_{1}=+\infty$.

Proof: Let $v(t, x)$ be an auxiliary function** which is smooth everywhere in the region $0 \leqq t<t_{1}$ except on the characteristic surfaces $C^{(p)}$ emanating from $\Gamma . v$ is to be so chosen that its jumps and those of its derivatives across these surfaces are equal to the unique solutions of the transport equations and initial conditions for $u$. Then the function $\Psi=L v$ is smooth; for by construction the function $L u$ and all of its derivatives are continuous across the $C^{(p)}$. Let $u=v+w$ where $w$ is a solution of the inhomogeneous equation

$$
L w=-\Psi .
$$

The initial conditions on $w$ are

$$
w(0, x)=g(x)-v(0, x) .
$$

By construction of $v$, both $\Psi=L v$ and $w(0, x)$ are smooth. Therefore, by Theorem 1 , the solution $w$ exists and is smooth. It follows that $u(0, x)=g(x)$ and $L u=0$ everywhere except on the surfaces $C^{(p)}$ where $u$ satisfies the transport equations. Now, as in (9) and (10),

$$
\iint u M \Omega d x d t=\iint \Omega L u d x d t+\sum_{p} \int_{C^{(p)}} \Omega H^{0(p)}[u]^{p} \alpha d S .
$$

But $L u=0$, and $H^{0(p)}[u]^{p}=0$. Hence (4) is satisfied. Thus it is proved that there exists a weak solution $u$.

For the uniqueness, let $u=u_{1}-u_{2}$, where $u_{1}$ and $u_{2}$ are solutions in $\kappa$. Then $u$ is in $\mathscr{T}$ and is a weak solution of the problem with $g(x) \equiv 0$. The discontinuities of $u$ and its first derivatives can occur only on characteristic surfaces. These always intersect the plane $t=0$. But the initial values for the transport equations on these surfaces are zero. Hence the discontinuities are zero. It follows that $u$ is continuously differentiable, so $u$ is a strong solution. Therefore,

\footnotetext{
${ }^{*} t_{1}$ is the smallest value of $t$ for which a caustic appears.

**The device of introducing this function and much of the foregoing construction are due to R. Courant \& P. Lax [3].
} 
from the uniqueness assertion of Theorem $1, u \equiv 0$. This proves the uniqueness and completes the proof of the theorem.

5. Initial-boundary value problems for symmetric hyperbolic equations. In addition to initial value problems for symmetric hyperbolic equations, we may also consider the so-called mixed, or initial-boundary value problems. If $\beta(x)=0$ is the equation of any smooth surface $B$ in the $x$-space, the transformation $z_{\nu}=\alpha_{\nu}(x), \nu=1, \cdots, n$, with $\alpha_{n}=\beta$, maps the surface $B$ into the plane $z_{n}=0$, while preserving the symmetric hyperbolic nature of $L$ and the smoothness of its coefficients. Thus without loss of generality we may take our boundary to be $x_{n}=0$. On this boundary we will require the solution $u$ to satisfy a certain type of homogeneous boundary condition* which we will now explain.

The vector $u$ is an element of a $k$-dimensional Euclidean vector space $V$. Consider a subspace $T$ of $V$ with the following properties:

1. The matrix $A^{n}$ is non-positive over $T$, i.e., $u A^{n} u \leqq 0$ for every $u$ in $T$.

2. $T$ is maximal, i.e., the dimension of $T$ is as large as the dimension of any subspace having property 1.

The boundary condition may now be expressed simply as

$$
u(x, t) \text { in } T \text { for } x_{n}=0 .
$$

If $T^{\perp}$ is the orthogonal complement of $T$, and $T^{\perp}$ is spanned by the vectors $b^{1}, \cdots, b^{m}$, the condition may also be stated as

$$
u b^{s}=0, \quad s=1, \cdots, m, \text { for } x_{n}=0 .
$$

Here $u b^{s}$ denotes the inner product. The vectors $b_{s}$ and the space $T$ need not be fixed but may be functions of the point on the boundary. We will take them to be smooth functions. For problems involving a boundary we will make use of the following generalization of Theorem 1 [6], [7]:

Theorem 1-B: Let $L$ be a symmetric hyperbolic operator with smooth coefficients; let $\phi(t, x)$ be a smooth function defined in the domain $D: t \geqq 0, x_{n} \geqq 0$; and let $g(x)$ be a smooth function defined in the domain $x_{n} \geqq 0$ which satisfies the boundary condition: $g$ in $T$, for $x_{n}=0$ and $t=0$. Then the initial-boundary value problem

$$
L u=\phi, \quad u(0, x)=g(x), \quad u \text { in } T, \text { for } x_{n}=0
$$

has a unique smooth solution $u(t, x)$ defined in $D$. The value of the solution $u$ at a given point depends on the values of the coefficients, the functions $\phi$ and $g$, and the boundary conditions only in a finite region.

Weak solutions will be defined as before, the only additional requirements

\footnotetext{
*In Maxwell's equations, the conditions on $E$ and $H$ at the surface of a perfect conductor can be shown to be conditions of this type. See Part II, Section 2.
} 
being the boundary condition (65) and the requirement that the region $R$ which is the support of the testing function $\Omega$ shall not intersect the half-space $x_{n} \leqq 0$.

The entire discussion in Sections $1,2,3$ now remains valid in the region $0 \leqq t<\min \left(t_{1}, t_{2}\right)$ where $t_{2}$ is the smallest value of $t$ for which one of the characteristic surfaces emanating from $\Gamma$ intersects the boundary. In order to extend our existence theorem beyond $t_{2}$ we must first extend our a priori calculation of the discontinuities of $u$. This is done in the next section.

6. Initial conditions for the transport equations at a boundary. Suppose that a characteristic surface $C$ emanating from the manifold $\Gamma$ in the initial hyperplane intersects the boundary $B$ in an $n-1$ dimensional manifold $\Delta$. $\Delta$ will be smooth in the region $t<t_{1}$, for there $C$ is smooth. We now consider all the characteristic surfaces which intersect $B$ in $\Delta$ and attempt to choose initial conditions for the transport equations on these surfaces in such a way that the jumps in $u$ and its derivatives will be compatible with the boundary conditions (65).

Let $\delta_{0}, \cdots, \delta_{n-1}, 0$ denote components of the unit normal to $\Delta$ in the plane $x_{n}=0$. Let $\phi^{0(p)}=0$ be the equation of a characteristic surface $C^{(p)}$ whose intersection with $B$ is $\Delta$. Then $\phi_{x_{\nu}}^{0(p)}=\rho \delta_{\nu}$ on $\Delta(\nu=0, \cdots, n-1)$, where $\rho$ is a proportionality factor. Here $\phi_{x_{n}}^{0(p)}$ is a solution of

$$
\operatorname{det} H^{0(p)}=\operatorname{det}\left(\sum_{\nu=0}^{n} \phi_{x_{\nu}}^{0(p)} A^{\nu}\right)=\operatorname{det}\left(\phi_{x_{n}}^{0(p)} A^{n}+\rho F\right)=0 \text { on } \Delta
$$

where

$$
F=\sum_{\nu=0}^{n-1} \delta_{\nu} A^{\nu}
$$

Equation (67) will, in general, have complex roots $\phi_{x_{n}}^{0(p)}$. For each distinct real root there will be a corresponding characteristic surface through $\Delta$. We assume that the manifold $\Gamma$ is bounded away from the boundary $B$. Then it is easily seen that there will be a point $P$ on $\Delta$ of minimum $t$, and that, at $P, \delta_{1}=\cdots=$ $\delta_{n-1}=0$. Hence, at $P, \delta_{0}=1$ and $F=I$. It follows from the continuity of $F$ that, in some neighborhood of $P, F$ is non-singular. We now impose a new condition on the problem, the

Shadow Condition: $F$ shall be non-singular throughout $\Delta$.

This condition, as suggested by its name, has an interesting geometric interpretation which will be explained below.

It follows from the shadow condition that $F$ is positive definite on $\Delta$. Therefore we may set $F=J J^{\prime}$ where $J$ is non-singular and has an inverse $S$. Let $G=$ $S A^{n} S^{\prime}$. Then $G$ is symmetric and we may write

$$
\left(G-\alpha_{p}\right) Q^{(p)}=0, \quad p=1, \cdots, k .
$$


Here the $Q^{(p)}$ are a complete set of orthogonal eigenvectors of $G$, which we may normalize by the condition

$$
Q^{(p)} S S^{\prime} Q^{(p)}=1, \quad p=1, \cdots, k .
$$

The eigenvalues $\alpha_{p}$ are real. Set $R^{(p)}=S^{\prime} Q^{(p)}, p=1, \cdots, k$. It follows from (70) that the $R^{(p)}$ are unit vectors. They are clearly linearly independent. From (69), $J\left(S A^{n} S^{\prime}-\alpha_{p}\right) J^{\prime} R^{(p)}=0$, or

$$
\left(A^{n}-\alpha_{p} F^{\prime}\right) R^{(p)}=0, \quad p=1, \cdots, k .
$$

For each non-zero $\alpha_{p}$, set $\phi_{x_{n}}^{0(p)}=-\rho / \alpha_{p}$; then

$$
H^{0(p)} R^{(p)}=\left(\phi_{x_{n}}^{0(p)} A^{n}+\rho F\right) R^{(p)}=0 \text { if } \alpha_{p} \neq 0 .
$$

Equation (72) expresses the fact that for each distinct non-zero $\alpha_{p}$, there is a distinct characteristic surface $C^{(p)}$ passing through $\Delta$, and $R^{(p)}$ is a null eigenvector of the associated matrix $H^{0(p)}$. Since we may have multiple roots $\alpha_{p}$, we may have several distinct $R^{(p)}$ associated with the same matrix $H^{0(p)}$. As we have seen earlier, the jump in $u$ across $C^{(p)},[u]^{p}$, can be expressed in terms of the associated null eigenvectors. Therefore

$$
[u]^{\Delta}=\sum_{a}[u]^{\alpha}=\sum_{p=1}^{k} \sigma_{p}^{0} R^{(p)} .
$$

Since we have used only the non-zero $\alpha_{p}$, we may assume that $\sigma_{p}^{0}=0$ in (73) if $\alpha_{p}=0$. (If $A^{n}$ is singular there will be zero eigenvalues $\alpha_{p}$. For these $\alpha_{p}$ we have solutions of (72) with $\rho=0$. In this case the boundary is itself a characteristic surface.) We apply the boundary condition in the form (66):

$$
\left[b^{\nu} u\right]^{\Delta}=b^{\nu}[u]^{\Delta}=\sum_{p=1}^{k} b^{\nu} R^{(p)} \sigma_{p}^{0}=0, \quad \nu=1, \cdots, m .
$$

Of course, some of the $\sigma_{p}^{0}$ are already determined (roughly speaking, those associated with a characteristic surface emanating from the initial plane or from the boundary at an earlier time). Our object will be to show that (74) can be solved uniquely for the undetermined $\sigma_{p}^{0}$ in terms of the determined ones. It is easy to see that $\sigma_{p}^{0}$ will (will not) have a preassigned value at a point on $\Delta$ if the ray at that point, oriented in the direction of increasing $t$, proceeds out of (into) the region $x_{n}>0$, i.e., if $\dot{x}_{n}=R^{(p)} A^{n} R^{(p)}$ is $<0(>0)$. (We shall see that the possibility $\dot{x}_{n}=0$ is precluded by the shadow condition.)

We digress to explain the significance of the shadow condition. Since the $R^{(p)}$ are linearly independent they may be made the columns of a non-singular matrix $R$. Then the matrix $R^{\prime} F R=M=\left(m_{p q}\right)$ has entries $m_{p q}=R^{(p)} F R^{(\alpha)}$. But $R^{(p)} F R^{(q)}=R^{(p)} T T^{\prime} R^{(q)}=Q^{(p)} Q^{(q)}=0$ if $p \neq q$. Hence $M$ is a diagonal matrix and $\operatorname{det} M=\prod_{p=1}^{k} R^{(p)} F R^{(p)}$. The shadow condition now guarantees that

$$
R^{(p)} F R^{(p)} \neq 0, \quad p=1, \cdots, k .
$$


From (71),

$$
R^{(a)} A^{n} R^{(p)}=\alpha_{p} R^{(q)} F R^{(p)}
$$

For all characteristic surfaces $C^{(p)}$ except the boundary itself $\alpha_{p} \neq 0$, hence $\dot{x}_{n}=R^{(p)} A^{n} R^{(p)} \neq 0$. But $\dot{x}_{n}=0$ if and only if the ray is tangent to the boundary. Thus the shadow condition suffices to ensure that no ray will be tangent to the boundary. In the language of physical applications, we say that there are no shadows in the problem.

Returning to our examination of the linear algebraic system (74), let $R^{(p)} A^{n} R^{(p)}$ be $>0$ for $p=1, \cdots, s$ and $\leqq 0$ for $p=s+1, \cdots, k$. We rewrite (74), placing known quantities on the right side:

$$
\sum_{p=1}^{s} b^{\nu} R^{(p)} \sigma_{p}^{0}=-\sum_{p=s+1}^{k} b^{\nu} R^{(p)} \sigma_{p}^{0}, \quad \nu=1, \cdots, m .
$$

Our object of proving that (77) has a unique solution will be attained if we can show that $s=m$ and the coefficient matrix $\left(b^{\nu} R^{(p)}\right)$ is non-singular. To this end, we shall employ some simple lemmas proved in Appendix 2. From (76), $R^{(p)} A^{n} R^{(p)}=0$ if $p \neq q$. Let $v=\sum_{p=1}^{s} c_{p} R^{(p)}$. Then $v A^{n} v=\sum_{p=1}^{s} c_{p}^{2} R^{(p)} A^{n} R^{(p)}>0$ unless $v=0$. Hence $R^{(1)}, \cdots, R^{(s)}$ span a subspace $T_{1}$ over which $A^{n}$ is positive. Similarly $R^{(s+1)}, \cdots, R^{(k)}$ span a subspace $T_{2}$ over which $A^{n}$ is non-positive. It follows from Lemmas 4 and 2 that $\operatorname{dim} T_{1} \leqq m$ and $\operatorname{dim} T_{2} \leqq k-m$ where $m$ is the number of positive eigenvalues of $A^{n}$. From Lemma $3, m$ is also the dimension of $T^{\perp}$ (which is as it is used in (66), (74), and (77)). But $\operatorname{dim} T_{1}+$ $\operatorname{dim} T_{2}=k$. Hence $\operatorname{dim} T_{1}=s=m$. Thus the coefficient matrix $\left(b^{v} R^{(p)}\right)$ of (77) is square. Suppose it were singular. Then there would exist non-trivial numbers $d_{p}$ such that $\sum_{p=1}^{m} d_{p} b^{\nu} R^{(p)}=0, \nu=1, \cdots, m$. Set $u=\sum_{p=1}^{m} d_{p} R^{(p)}$. Then $u b^{\nu}=0, \nu=1, \cdots, m$, and $u A^{n} u=\sum_{p=1}^{m} d_{p}^{2} R^{(p)} A^{n} R^{(p)}>0$. But this contradicts the boundary conditions (see Section 5). Hence the coefficient matrix is non-singular and the solution of (77) exists and is unique. That solution $\sigma_{1}^{0}, \cdots, \sigma_{m}^{0}$ provides the initial values for the transport equation of order zero.

To obtain the initial conditions for the higher transport equations we proceed as follows: Let $s$ be a unit vector in the ray direction, and $\partial / \partial s$ denote differentiation in that direction. Now $s$ and $y_{0}$, being orthogonal directions, determine a two-dimensional plane which must intersect the ( $n$ dimensional) boundary in a direction $N$ which lies in the boundary, but which cannot lie in $\Delta$. This is true because $y_{0}$ is orthogonal to $\Delta$ and the shadow condition guarantees that $s$ is not in $\Delta$ (see Fig. 2). We now differentiate the boundary condition (66), in the direction $N, r$ times to obtain

$$
u_{N^{r}} b^{\nu}+r u_{N^{(r-1)}} b_{N}^{\nu}+\cdots=0 \text { on } x_{n}=0, \quad \nu=1, \cdots, m,
$$

or

$$
\left[u_{N^{r}}\right]^{\Delta} b^{\nu}=-r\left[u_{N^{(r-1)}}\right]^{\Delta} b_{N}^{\nu}-\cdots \quad \text { on } \quad x_{n}=0, \quad \nu=1, \cdots, m .
$$




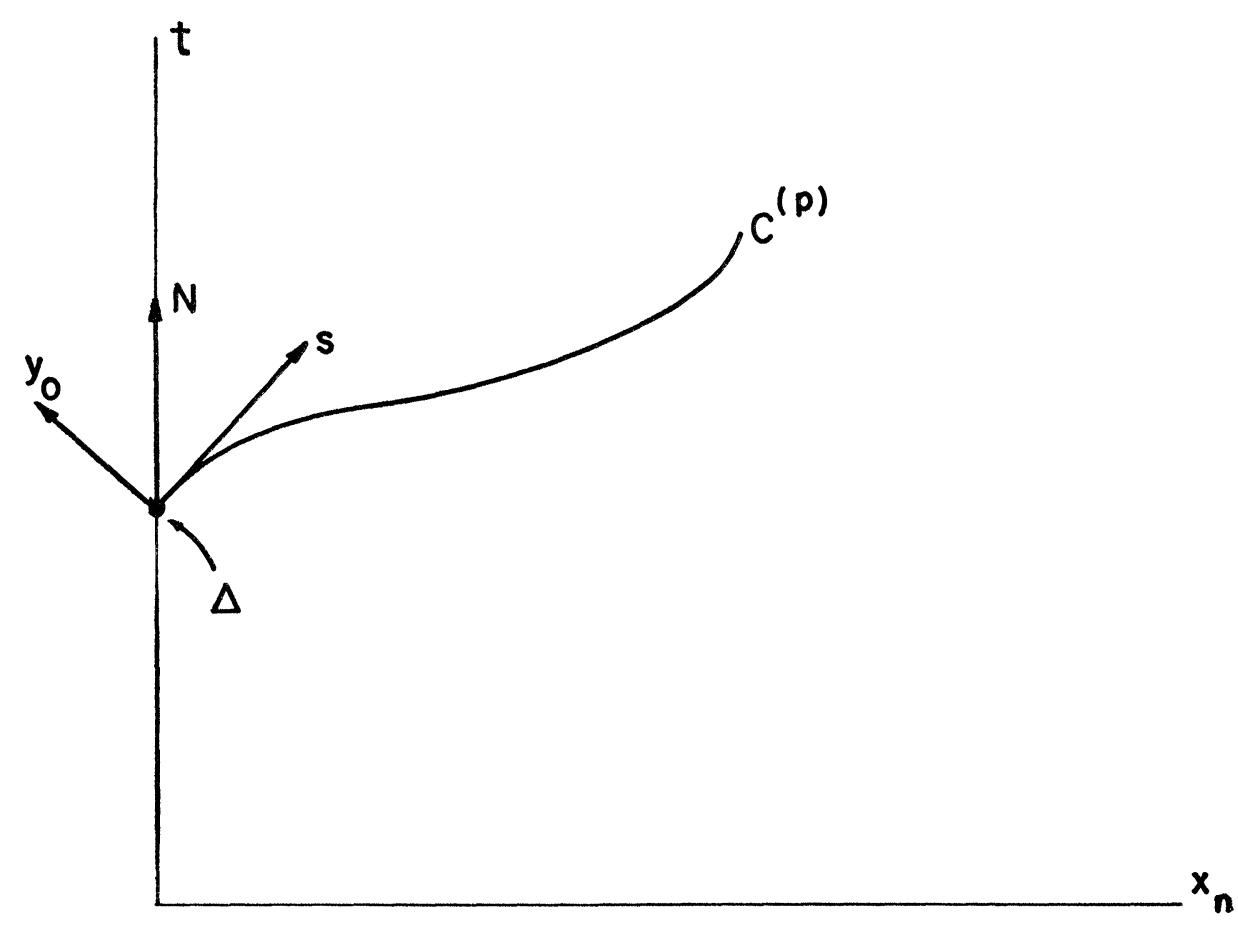

FIGURE 2

Relative orientations of the directions $y_{0}, N, s$ at the boundary.

As in Section 2, we express $u_{N^{r}}$ in terms of derivatives in the $y_{0}$ and $s$ directions:

$$
u_{N^{r}}=\sum_{\nu=0}^{r} e_{\nu}^{p} u_{\nu_{0}(r-p)_{8} v}
$$

or

$$
\left[u_{N^{r}}\right]^{p}=e_{0}^{p}\left[u_{\nu_{0} r}\right]^{p}+\sum_{\nu=1}^{r} e_{\nu}^{p}\left[u_{y_{0}(r-\nu)}\right]_{s^{\nu}}^{p}
$$

It is important to note that the shadow condition guarantees that the directions $s$ and $N$ do not coincide, hence $e_{0}^{p} \neq 0$. Suppose now that $\left[u_{y_{0} \rho}\right]^{p}$ has been determined for each characteristic surface $C^{(p)}$ which intersects the boundary in the manifold $\Delta$, for $\rho=0,1, \cdots, r-1$. Then the jumps in all derivatives of order less than $r$ are known. To avoid unnecessary writing, let $W_{j}$ denote known quantities. Then

$$
\left[u_{N^{r}}\right]^{\Delta}=\sum_{p}\left[u_{N^{r}}\right]^{p}=\sum_{p} e_{0}^{p}\left[u_{y_{0} r}\right]^{p}+W_{1} .
$$

From (79)

$$
\sum_{p} e_{0}^{\nu}\left[u_{\nu_{0}}\right]^{p} b^{\nu}=W_{2}, \quad \nu=1, \cdots, m
$$


On each $C^{(p)},\left[u_{y_{0}}\right]^{p}$ can be expressed in terms of the eigenvectors of $H^{0(p)}$, the coefficients of the eigenvectors which are not null being known from (23). The totality (for all $C^{(p)}$ ) of the null eigenvectors are simply the $R^{(p)}$, $p=1, \cdots, k$. Furthermore, the coefficients of $R^{(m+1)}, \cdots, R^{(k)}$ are known; hence

$$
\sum_{p=1}^{m} b^{\nu} R^{(p)} e_{0}^{p} \sigma_{p}^{r}=W_{3}, \quad \nu=1, \cdots, m .
$$

Comparison with (77) now shows that (84) can be solved uniquely for the quantities $e_{0}^{p} \sigma_{p}^{r}, p=1, \cdots, m$, hence, since $e_{0}^{p} \neq 0$, for the $\sigma_{p}^{r}$, which provide the initial values for the transport equation of order $r$.

7. Existence and uniqueness theorem for the discontinuous initial boundary value problem. In order to generalize the theorem of Section 3 to problems with boundaries we need only impose upon the auxiliary function $v$ the additional requirement that it satisfy the boundary condition. This can be done because the discontinuities across the characteristic surfaces which intersect the boundary are such as to be compatible with the boundary conditions. Now by Theorem $1-\mathrm{B}$ we can require that the function $w$ also satisfy the boundary condition. Then the function $u=v+w$ will satisfy the boundary condition. The uniqueness argument requires an obvious modification. Thus we have

Theorem 2-B: Let the initial boundary value problem

$$
\begin{gathered}
L u=0, \\
u(0, x)=g(x), \\
u(x, t) \text { in } T, \text { for } x_{n}=0,
\end{gathered}
$$

be given. Here $L$ is a symmetric hyperbolic operator with smooth coefficients defined in the domain $D: t \geqq 0, x_{n} \geqq 0 ; g(x)$ is smooth on either side of a smooth $n-1$ dimensional manifold $\Gamma$ in the plane $t=0$; while $g$ and its derivatives have jump discontinuities across $\Gamma$ and $g$ satisfies the boundary condition: $g$ in $T$ for $x_{n}=0$ and $t=0$. Let the multiplicity and shadow conditions be satisfied. Then there exists one and only one solution $u$ in the class $\mathcal{K}$ in the region $0 \leqq t<t_{1}, x_{n} \geqq 0$. $u$ is smooth everywhere in that region except on the characteristic surfaces emanating from $\Gamma$ and from the intersections $\Delta$ (which may be multiple*) of such surfaces with the boundary $x_{n}=0$. Across these surfaces, $u$ and its derivatives suffer jump discontinuities which satisfy the transport equations. If the caustic condition is satisfied we may take $t_{1}=+\infty$.

Note. By virtue of the remarks made at the beginning of Section 5 the above theorem is immediately valid for any domain $D$ which can be mapped by a

\footnotetext{
*It is clear that our construction and hence this theorem are valid for multiple "reflections" from the boundary.
} 
smooth transformation of the space coordinates onto the domain $t \geqq 0, x_{n} \geqq 0$.* The theorem is also valid for more general domains such as the interior of a cylinder with generators parallel to the $t$-axis or domains bounded by several smooth surfaces. In these cases we must employ the analogues of Theorem 1-B valid for such domains [6], [7].

\section{Appendix I}

Lemma: Let $P, A$ be real symmetric matrices of dimension $k ;$ let $M(\zeta)=P+\zeta A$ have eigenvalues whose multiplicities are the same for every real value of $\zeta$. Let $\lambda(\zeta)$ be an eigenvalue of $M$ with associated orthonormal eigenvectors $R^{1}(\zeta), \cdots, R^{\alpha}(\zeta)$. (Thus $q$ is independent of $\zeta$.) Then $\lambda(\zeta)$ is an analytic function of $\zeta$ in a neighborhood of every real point $\zeta$, and the $R^{\nu}(\zeta)$ may be so chosen as to have the same property.

Proof: $\lambda$ is a solution of the equation

$$
\operatorname{det}(\lambda I-M)=G(\lambda, \zeta)=\lambda^{k}+g_{(k-1)}(\zeta) \lambda^{k-1}+\cdots+g_{0}(\zeta)=0 .
$$

Here the $g_{\nu}$ are polynomials in $\zeta$. Let us factor $G$ into irreducible polynomials $H$ of the form

$$
H(\lambda, \zeta)=h_{p}(\zeta) \lambda^{p}+h_{(p-1)}(\zeta) \lambda^{p-1}+\cdots+h_{0}(\zeta)
$$

Then each eigenvalue $\lambda$ is a solution of an equation

$$
H(\lambda, \zeta)=0 .
$$

Thus $\lambda$ is by definition an algebraic function of $\zeta$. From the theory of algebraic functions, $\dagger$ we know that the equation $H\left(\lambda, \zeta_{0}\right)=0$ has $p$ distinct roots except at certain exceptional points $\zeta_{0}$ where either

1. $h_{p}$ vanishes

or

2. the discriminant of the equation, which is a polynomial in $\zeta$, vanishes. The discriminant of an irreducible polynomial cannot vanish identically. Hence the singular points of type 2 are finite in number. Furthermore, in our case there can be no real points of type 2 ; for at such points the multiplicity of some eigenvalue must be greater than at neighboring points. Also we observe that $h_{p}(\zeta)$ must be a constant, so there can be no points of type 1 . Since algebraic functions are analytic except at exceptional points, the assertion for $\lambda(\zeta)$ follows.

*It should be noted that the mapping induces a change in the statement of the boundary condition and the shadow condition. Thus, e.g., if $\beta(x)=0$ defines the boundary, the boundary condition becomes $u(x, t)$ in $T$ for $\beta(x)=0$, where $T$ is the maximal subspace such that the matrix $\sum_{\nu=1}^{n} \beta_{x_{\nu}} A^{\nu}$ is non-positive over $T$.

†See e.g., [8] for the statements about algebraic functions which we employ here. 
The eigenvectors $R$ are solutions of a linear algebraic system

$$
T R=0 .
$$

Here $T=\left(t_{i j}\right)=\lambda(\zeta) I-P-\zeta A$. Thus the $t_{i j}$ are analytic functions of $\zeta$ for real $\zeta$. The nullity of $T$ is $q$, and if $r$ denotes the rank of $T$, we have

$$
r+q=k \text {. }
$$

To solve (4) for the components of $R$ we may first reduce it to an equivalent system of $r$ equations and $k$ unknowns. We then assign arbitrary values to $k-r=q$ of the unknowns $x_{1}, \cdots, x_{q}$ such that the remaining $r \times r$ coefficient matrix is non-singular. We may then solve for the remaining $r$ unknowns by Cramer's rule. The $x_{q+1}, \cdots, x_{k}$ will therefore be rational functions of the $x_{1}, \cdots, x_{a}$ and the $t_{i j}$ with non-vanishing denominator. By choosing $x_{1}, \cdots, x_{a}$ as analytic functions of $\zeta$ we are guaranteed that $R$ is an analytic function of $\zeta$. We obtain $q$ linearly independent solutions $R(\zeta)$, and the analyticity is preserved in the orthonormalization procedure to obtain the $R^{1}(\zeta), \cdots, R^{\alpha}(\zeta)$.

\section{Appendix 2}

Definitions: Given a $k$-dimensional vector space $V$, a real symmetric operator $A$, and a subspace $T, A$ is non-positive over $T$ if $(u, A u) \leqq 0$ for all $u$ in $T$. $A$ is positive over $T$ if $(u, A u)>0$ for all $u \neq 0$ in $T$. Let $\alpha_{\nu}, r^{\nu}$ denote (real) eigenvalues and orthonormal eigenvectors of $A$; i.e., $A r^{\nu}=\alpha_{\nu} r^{\nu}, \nu=1, \cdots, k$. Let $\alpha_{\nu}>0$ for $\nu=1, \cdots, m, \alpha_{\nu} \leqq 0$ for $\nu=m+1, \cdots, k$.

Lemma 1: Let T, $R$ be disjoint subspaces of $V$ (i.e., if $u$ is in $T$ and in $R$ then $u=0)$. Then $\operatorname{dim} T+\operatorname{dim} R \leqq k$.

Proof: The proof of this lemma is trivial.

Lemma 2: Let $A$ be non-positive over $T$. Then $\operatorname{dim} T \leqq k-m$.

Proof: Let $R$ be the subspace spanned by $r_{1}, \cdots, r_{m}$. Then $T$ and $R$ are disjoint, for if $u$ is in $R$ and in $T$, we have

$$
u=\sum_{\nu=1}^{m} c_{\nu} r^{\nu}, \quad A u=\sum c_{\nu} \alpha_{\nu} r^{\nu}, \quad(u, A u)=\sum c_{\nu}^{2} \alpha_{\nu} \leqq 0 .
$$

Hence $c_{\nu}=0, \nu=1, \cdots, m$. Therefore $u=0$.

Lemma 3: Let $T$ be a subspace of $V$ of maximal dimension such that $A$ is nonpositive over $T$. Then $\operatorname{dim} T=k-m$.

Proof: Let $T_{1}$ be the subspace spanned by $r_{m+1}, \cdots, r_{k}$. Then $A$ is nonpositive over $T_{1}$ and $\operatorname{dim} T_{1}=k-m$.

Lemma 4: Let $A$ be positive over $P$. Then $\operatorname{dim} P \leqq m$. 
Proof: Let $T_{1}$ be defined as above. Then $T_{1}$ and $P$ are disjoint; for if $u \neq 0$ is in $T_{1}$ and in $P$, we have

$$
u=\sum_{\nu=m+1}^{k} c_{\nu} r^{\nu}, \quad A u=\sum c_{\nu} \alpha_{\nu} r^{\nu}, \quad(u, A u)=\sum c_{\nu}^{2} \alpha_{\nu}>0,
$$

which is a contradiction.

\section{AcKNOWLedgment}

The author wishes to express his appreciation to the members of the faculty and staff of the Institute of Mathematical Sciences for their advice and criticism. In particular, he is indebted to Professor Joseph B. Kellenf or his assistance and encouragement during the preparation of this paper.

\section{References}

[1] Courant, R. \& Hilbert, D., Methoden der Mathematischen Physik; Vol. II, Chapters V, VI, Springer, Berlin, 1937.

[2] Keller, J. B., Geometrical acoustics I. The theory of weak shock waves; J. Appl. Phys., 25, 938-947, 1954.

[3] Courant, R. \& Lax, P. D., The propagation of discontinuities in wave motion; Proc. of the Nat'l. Acad. of Sci., 42, No. 11, 872-876, Nov., 1956.

[4] Friedrichs, K. O., Symmetric hyperbolic linear differential equations; Comm. Pure and and Appl. Math., 7, No. 2, 345-393, May, 1954.

[5] LAX, P. D., On Cauchy's problem for hyperbolic equations and the differentiability of solutions of elliptic equations; Comm. Pure and Appl. Math., 8, No. 4, 615-633, Nov., 1955.

[6] FrIedrichs, K. O., Differential equations without regard to type; unpublished.

[7] Lax, P. D., Mixed problems for symmetric hyperbolic systems; unpublished.

[8] KNopp, K., Theory of Functions. Part II, Chapter 5, Dover, N. Y., 1947.

Institute of Mathematical Sciences

New York University 